Fadeyi et al., Afr. J. Infect. Dis. (2016) 10 (1): 32 - 37

http://dx.doi.org/10.4314/ajid.v10i1.7

\title{
PREVALENCE AND ANTIBIOTIC SUSCEPTIBILITY PATTERN OF ESBL PRODUCING KLEBSIELLAE ISOLATED FROM CLINICAL SPECIMENS IN A NIGERIAN TERTIARY HOSPITAL
}

\author{
${ }^{1}$ Fadeyi A; ${ }^{1}$ *Zumuk C. P; ${ }^{2}$ Raheem R.A; ${ }^{1}$ Nwabuisi C; ${ }^{3}$ Desalu O. O.
}

\author{
${ }^{1}$ Department of Medical Microbiology and Parasitology, University of Ilorin, Ilorin, Nigeria, ${ }^{2}$ Department of Medical \\ Microbiology and Parasitology, University of Ilorin Teaching Hospital, Ilorin, Nigeria, ${ }^{3}$ Department of Medicine, \\ University of Ilorin, Ilorin, Nigeria \\ *E-mail: prisgee@yahoo.com
}

\begin{abstract}
Background: Infection by Extended Spectrum Beta Lactamases (ESBLs) producing bacteria is a threat to man as a consequence of treatment challenges. This study evaluated the prevalence and antimicrobial susceptibility pattern of ESBL producing Klebsiellae (EPK) in clinical specimens at the University of Ilorin Teaching hospital, Ilorin (UITH), Nigeria.

Methods: ESBL production was assayed using Double Discs Synergy Test (DDST). Antimicrobial susceptibility was performed by Modified KirbyBaeur method with the organism tested against ceftazidime $(30 \mu \mathrm{g})$, cefotaxime $(30 \mu \mathrm{g})$, amoxicillin-clavulinic acid $(20 / 10 \mu \mathrm{g})$, cefepime $(30 \mu \mathrm{g})$, ciprofloxacin $(5 \mu \mathrm{g})$, gentamicin $(10 \mu \mathrm{g})$, trimethoprim-sulphamethoxazole $(23.75 / 1.25 \mu \mathrm{g})$, imipenem $(10 \mu \mathrm{g})$ and doripenem $(10 \mu \mathrm{g})(\mathrm{Oxoid}, \mathrm{UK})$.

Results: Fifty (26.7\%) of the 187 Klebsiellae studied were EPK comprising of 37(26.8\%) Klebsiella pneumoniae and 13(26.5\%) Klebsiella oxytoca. EPK were mostly from wound specimens (24.0\%) although Klebsiellae were mostly occurring in sputum (26.2\%). The EPK were resistant to ceftazidime $(100 \%)$, cefotaxime $(94.0 \%)$, trimethoprim-sulphamethoxazole $(92.0 \%)$, gentamicin $(70.0 \%)$ and ciprofloxacin $(70.0 \%)$ but $100 \%$ susceptible to both doripenem and imipenem.

Conclusion: The prevalence of EPK in this study is high and they are multi-drug resistant. Carbapenems are the best antibiotic treatment option for infections arising from these organisms although a coordinated rational usage is desired along with functional antibiotic prescription policy to avoid treatment failures. Continuous surveillance for ESBL producing Klebsiellae and resistance monitoring are necessary routine to strengthen infection control policies.
\end{abstract}

Keywords; ESBL, cephalosporins, Klebsiella, antibiotics susceptibility, drug resistance.

\section{Introduction}

Klebsiella species and Escherichia coli have been reported to harbor ESBL enzyme with $K$. pneumoniae being the most frequent isolate (Paterson and Bonomo, 2005). Klebsiella spp are involved in various infections affecting both immunocompetent and immunocompromised hosts with a wide spectrum involving urinary, respiratory and gastro intestinal tracts. In addition, they cause bacteremia, septicaemia, and various organ and soft tissue infections. ESBL producing Klebsiella spp have emerged as a major problem in hospitalized as well as community based patients as a result of the difficulty it poses to treatment (Paterson et al., 2003). Extended Spectrum Beta Lactamase (ESBL) producing organisms breakdown penicillins and cephalosporins, through the production of $\beta$-lactamases capable of hydrolyzing the $\beta$-lactam ring of these drugs (Bradford, 2001). The first ESBL isolates were discovered in Germany in the mid-1980s and subsequently in the United States of America in the late 1980s shortly after the introduction of ceftazidime and cefotaxime into clinical practice (Knothe et al., 1983). The emergence and spread of ESBL producing organisms is believed to be due to the dissemination of clones of some epidemic strains as well as horizontal transmission of resistance gene-carrying plasmids among bacterial organisms (Rodriquez-Bano et al., 2004). The rise in the use of second and third generation cephalosporins to treat infections have similarly led to the development and selection of multiple drug resistant bacteria like ESBL producers (Pitout and Laupland, 2008). These organisms often acquire resistance to non- $\beta$-lactam antibiotics including aminoglycosides and fluoroquinolones, resulting in multi-drug resistant properties.

Risk factors that have been associated with infection or colonization with ESBL producing organisms including Klebsiella strains are prolonged hospitalization, stay in nursing homes, use of invasive devices (e.g., urinary catheters, central venous lines, and endotracheal tubes), and use of antibiotics belonging to third-generation cephalosporins, fluoroquinolones, and aminoglycosides group (Paterson, 2000). The burden of antibiotic resistance including the ESBL phenotypes cannot be over- emphasized especially in neonates, children and the immune -compromised as it leads to an increased risk of death, length of stay in hospitals and cost of care (Schwaber M et al., 2006 ).

In recent times, EPK showing multi-drug resistance features are being isolated in many laboratories. It is therefore necessary to search in every setting for this organism which has constituted itself into a threat to man consequent upon its treatment challenges. It is similarly important to define the best choice of antibiotic therapy for the infections caused by EPK with the sole aim of reducing the length of patient stay in hospitals, cost of treatment and rates of mortality and morbidity which is the primary focus of this study.

\section{Materials and Methods}

This study was done at the UITH Medical Microbiology laboratory. Ethical clearance was obtained from the Ethical committee of the institution. One hundred and eighty seven (187) clinical isolates of Klebsiella were obtained non-repetitively from different clinical specimens including blood, sputum, wound, urine, ear, eye and throat swabs between October, 2013 and February, 2014. . 
Fadeyi et al., Afr. J. Infect. Dis. (2016) 10 (1): 32 - 37

\section{http://dx.doi.org/10.4314/ajid.v10i1.7}

\section{Re- characterization and Storage of Isolates}

The organisms were re-characterized using standard microbiological techniques (Cheesebrough, 2006). Identified Klebsiella isolates were stored at $-20^{\circ} \mathrm{C}$ using $20 \%$ Brain heart infusion (BHI)-glycerol broth until further processed.

\section{ESBL Detection}

ESBL production was assayed by the double disc synergy test (DDST) method. Briefly, $30 \mu \mathrm{g}$ of ceftazidime dics (Oxoid, UK) was placed at a distance of $20 \mathrm{~mm}$ (edge to edge) from an amoxicillin-clavulinic acid disc (20/10 $\mu \mathrm{g}$, Oxoid, UK) on Mueller-Hinton agar plate already inoculated with the test organism. Inoculum was prepared by suspending the test organism in sterile physiologic saline inside Bijou bottle and adjusting its turbidity to that of $0.5 \mathrm{McFarland}$ standards. Inoculation was done by rolling the cotton swabs, previously dipped into the inoculum suspension with the excess fluid removed by compressing the swab against the inside wall of the container, on the surface of the Mueller-Hinton agar. The set-up was incubated at $35^{\circ} \mathrm{C}$ for $16-18$ hours. (CLSI, 2013) Escherichia coli ATCC 25922 was used as negative control. Positive test consisted of enhancement/amplification of inhibition zone around ceftazidime and towards amoxicillin/clavulinic acid disc giving a dump-bell appearance.

\section{Antimicrobial Susceptibility testing}

This was done using the modified Kirby Baeur-Method. Inoculum preparation, inoculation and incubation were done as described under

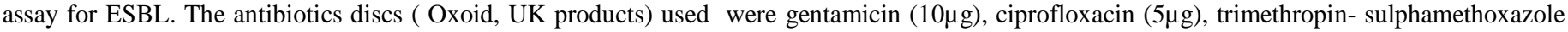
$(1.25 / 23.75 \mu \mathrm{g})$, cefepime $(30 \mu \mathrm{g})$, amoxicillin-long clavulinic acid $(20 / 10 \mu \mathrm{g})$, cefotaxime $(30 \mu \mathrm{g})$, ceftazidime $(30 \mu \mathrm{g})$, imipenem $(10 \mu \mathrm{g})$ and doripenem $(10 \mu \mathrm{g})$. The discs were placed on the inoculated Mueller-Hinton agar at a distance of $15 \mathrm{~mm}$ from the edge of an inoculated plate and about $25 \mathrm{~mm}$ from disc to disc. A maximum of 6 discs were placed on a $90 \mathrm{~mm}$ petri dish. Within 30mins of applying the discs, the plates were inverted and incubated aerobically at $35^{\circ} \mathrm{C}$ for 16- 18hours. Escherichia coli ATCC 25922 was used as control. Zones of inhibition were recorded with a meter rule and results were interpreted as sensitive (S), intermediate (I) and resistant (R) according to CLSI standard.

\section{Data analysis}

Data were entered into the computer using excel spread sheet and statistical analysis was performed using Microsoft excel (2007) and the Statistical Product and Service Solutions (SPSS) software version 19.0 (IBM SPSS, 2012).

\section{Results}

Of the 187 Klebsiella isolates examined, 138 (73.8\%) were Klebsiella pneumoniae and the remainder Klebsiella oxytoca, 49(26.2\%). In all, 50 (26.7\%) of the Klebsiella isolates were EPK comprising of 37(74\%) Klebsiella pneumoniae and 13(26\%) Klebsiella oxytoca (Table 1).

Table 2 shows the distribution of EPK according to specimens while table 3 shows distribution according to wards. Klebsiella isolates (26.2\%) were mostly isolated from sputum but EPK were mostly found in wound culture specimens (24.0\%). Majority (75\%) of the Klebsiella isolates from the ICU were EPK.

Table 4 showed the antimicrobial susceptibility pattern of EPK. The EPK were multi-drug resistant being resistant to ceftazidime (100\%), cefotaxime (94.0\%), and trimethoprim-sulphamethoxazole (92.0\%) among others. They were largely susceptible to carbapenems displaying no resistance against doripenem $(0 \%)$ and imipenem $(0 \%)$ but one isolate is intermediately susceptible to imipenem.

Figure 1 shows the dump-bell shaped inhibition zone around ceftazidime and towards amoxicillin-clavulinic acid disc which is characteristic of ESBL producers.

Table 1: Prevalence of ESBL producing Klebsiella spp

\begin{tabular}{|c|c|c|}
\hline Organism & Total Number (\%) & Number of ESBL Producers (\%) \\
\hline Klebsiella oxytoca & $49(26.2)$ & $13(26)$ \\
\hline Klebsiella pneumoniae & $138(73.8)$ & $37(74)$ \\
\hline Total & $\mathbf{1 8 7}(\mathbf{1 0 0})$ & $\mathbf{5 0}(\mathbf{1 0 0})$ \\
\hline
\end{tabular}

\section{Discussion}

The prevalence of EPK in this study was 26.7\%. This result was similar to the study done in Enugu (Ejikeugwu et al., 2013) Nigeria, and that of Iran (Shanyanfar et al., 2010) where prevalence rates of $26.0 \%$ and $27.0 \%$ were obtained respectively. A slightly higher prevalence of $36.6 \%$ was obtained in Benin (Ozazuwa and Ozazuwa, 2013). Much higher prevalence rates of $43.2 \%$ in Ibadan (Okesola and Oni, 2012), and 51.3\% in Ile Ife (Olowe et al., 2012), all in Nigeria, have been documented.

The high rates of EPK being globally reported is a major concern not only to healthcare givers but the general populace. EPK infections have impacted negatively on mankind constituting a special health and economic burden due to increased morbidity and mortality, increase in hospital stay and cost of treatment (Okonko et al., 2009a). High prevalence of ESBL production by bacteria has been associated with the irrational/ 
Fadeyi et al., Afr. J. Infect. Dis. (2016) 10 (1): 32 - 37

http://dx.doi.org/10.4314/ajid.v10i1.7

overuse of penicillins and cephalosporins in our environment (Akande et al., 2009) indicating the need for development and enforcement of an antibiotic prescription policy. Continuous surveillance through well-equipped laboratories for prompt accurate detection and reporting of EPK should also become a routine in our hospitals as a guide to functional infection control interventions.

Table 2: Specimen distribution of ESBL producing Klebsiella spp

\begin{tabular}{|c|c|c|}
\hline Specimen & Klebsiella Isolates collected & ESBL producers $\mathrm{n}(\%)$ \\
\hline Aspirate & $13(7.0)$ & $7(14.0)$ \\
\hline Blood & $17(9.1)$ & $4(8.0)$ \\
\hline Ear swab & $6(3.2)$ & $1(2.0)$ \\
\hline Eye swab & $4(2.1)$ & $1(2.0)$ \\
\hline Lochia & $1(0.5)$ & $1(2.0)$ \\
\hline Pus & $4(2.1)$ & $2(4.0)$ \\
\hline Sputum & $49(26.2)$ & $6(12.0)$ \\
\hline Throat swab & $2(1.1)$ & $2(4.0)$ \\
\hline Urine & $28(15.0)$ & $6(12.0)$ \\
\hline Urine catheter tip & $6(3.2)$ & $3(6.0)$ \\
\hline UVC tip & $13(7.0)$ & $5(10.0)$ \\
\hline Wound aspirate & $1(0.5)$ & $0(0)$ \\
\hline Wound biopsy & $1(0.5)$ & $0(0)$ \\
\hline Wound swab & $42(22.5)$ & $12(24.0)$ \\
\hline Total & $187(100)$ & $50(100)$ \\
\hline
\end{tabular}

Table 3: Ward Distribution of ESBL producing Klebsiella isolates

\begin{tabular}{lll}
\hline Ward & Isolates collected, $\mathbf{n}(\boldsymbol{\%})$ & ESBL producers, $\mathbf{n}(\boldsymbol{\%})$ \\
\hline A/E & $35(18.7)$ & $7(14.0)$ \\
CHEST & $1(0.5)$ & $1(2.0)$ \\
DENTAL & $1(0.5)$ & $1(2.0)$ \\
ENT & $3(1.6)$ & $1(2.0)$ \\
EPU & $12(6.4)$ & $1(2.0)$ \\
EYE & $1(0.5)$ & $0(0)$ \\
GYNAE & $12(6.4)$ & $4(8.0)$ \\
ICU & $4(2.1)$ & $3(6.0)$ \\
MW & $22(11.8)$ & $7(14.0)$ \\
NICU & $19(10.2)$ & $6(12.0)$ \\
OPD & $31(16.6)$ & $3(6.0)$ \\
STROKE & $6(3.2)$ & $4(8.0)$ \\
SW & $21(11.2)$ & $7(14.0)$ \\
OTHERS & $19(10.2)$ & $5(10.0)$ \\
\hline Total & $\mathbf{1 8 7}(\mathbf{1 0 0})$ & $\mathbf{5 0}(\mathbf{1 0 0})$ \\
\hline
\end{tabular}

A/E- Accident and Emergency, ENT- Ear Nose and Throat, EPU-Emergency Paediatric Unit, ICU-Intensive Care Unit, MW-Medical Ward, NICU-Neonatal Intensive Care Unit, OPD-Out-Patient Dept, SW-Surgical ward 
Fadeyi et al., Afr. J. Infect. Dis. (2016) 10 (1): 32 - 37

http://dx.doi.org/10.4314/ajid.v10i1.7

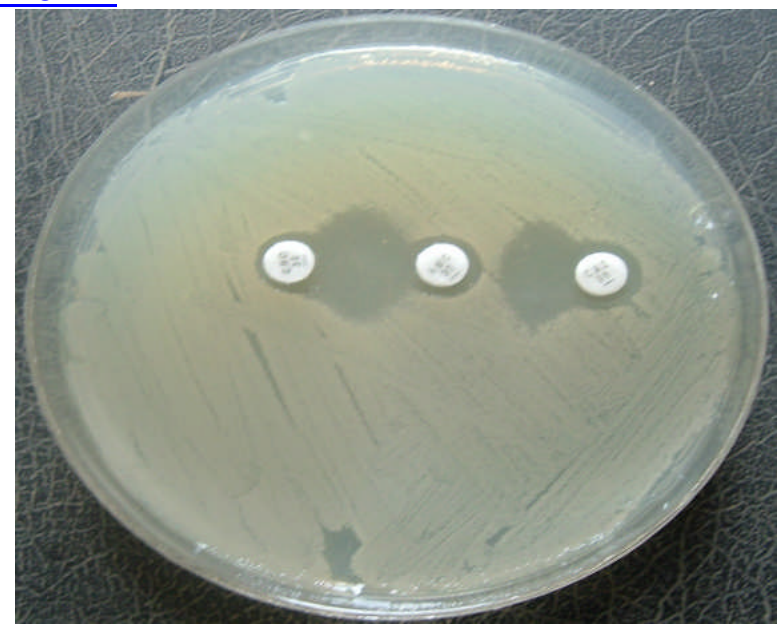

Figure 1: Dump-bell inhibition zone of positive DDST characteristic of ESBL producing Klebsiellae

Table 4: Antibiotic Susceptibility pattern of ESBL producing Klebsiella spp

\begin{tabular}{|c|c|c|c|c|}
\hline \multirow{2}{*}{ Antibiotics } & & \multicolumn{3}{|c|}{ ESBL producers. $n=50$} \\
\hline & & $\begin{array}{l}\text { Klebsiella spp } \\
n=50\end{array}$ & $\begin{array}{l}\text { K.oxytoca, } \\
n=13\end{array}$ & $\begin{array}{l}\text { K.pneumoniae, } \\
n=37\end{array}$ \\
\hline \multirow[t]{3}{*}{ Cefotaxime } & S (\%) & 0 & 0 & 0 \\
\hline & $\mathrm{I}(\%)$ & 0 & 0 & 0 \\
\hline & $\mathrm{R}(\%)$ & 100 & 100 & 100 \\
\hline \multirow[t]{3}{*}{ Ceftazidime } & $\mathrm{S}(\%)$ & 0 & 0 & 0 \\
\hline & I (\%) & 6.0 & 0 & 8.1 \\
\hline & $\mathrm{R}(\%)$ & 94.0 & 100 & 91.9 \\
\hline \multirow[t]{3}{*}{ Amox-clav } & $\mathrm{S}(\%)$ & 6.0 & 7.7 & 5.4 \\
\hline & $\mathrm{I}(\%)$ & 28.0 & 38.5 & 24.3 \\
\hline & $\mathrm{R}(\%)$ & 66.0 & 53.8 & 70.3 \\
\hline \multirow[t]{3}{*}{ Cefepime } & $\mathrm{S}(\%)$ & 30.0 & 15.4 & 35.1 \\
\hline & $\mathrm{I}(\%)$ & 50.0 & 61.5 & 45.9 \\
\hline & $\mathrm{R}(\%)$ & 20.0 & 23.1 & 18.9 \\
\hline \multirow[t]{3}{*}{ Gentamicin } & $\mathrm{S}(\%)$ & 20.0 & 7.7 & 24.3 \\
\hline & $\mathrm{I}(\%)$ & 10.0 & 15.4 & 8.1 \\
\hline & $\mathrm{R}(\%)$ & 70.0 & 76.9 & 67.6 \\
\hline \multirow[t]{3}{*}{ Ciprofloxacin } & $\mathrm{S}(\%)$ & 14.0 & 7.7 & 16.2 \\
\hline & $\mathrm{I}(\%)$ & 16.0 & 7.7 & 18.9 \\
\hline & $\mathrm{R}(\%)$ & 70.0 & 84.6 & 64.9 \\
\hline \multirow[t]{3}{*}{ TMP-SMX } & $\mathrm{S}(\%)$ & 6.0 & 0 & 8.1 \\
\hline & $\mathrm{I}(\%)$ & 2.0 & 0 & 2.7 \\
\hline & $\mathrm{R}(\%)$ & 92.0 & 100 & 89.2 \\
\hline \multirow{3}{*}{ Imipenem } & S (\%) & 98.0 & 100 & 97.3 \\
\hline & I $(\%)$ & 2.0 & 0 & 2.7 \\
\hline & $\mathrm{R}(\%)$ & 0 & 0 & 0 \\
\hline \multirow[t]{3}{*}{ Doripenem } & $\mathrm{S}(\%)$ & 100 & 100 & 100 \\
\hline & $\mathrm{I}(\%)$ & 0 & 0 & 0 \\
\hline & $\mathrm{R}(\%)$ & 0 & 0 & 0 \\
\hline
\end{tabular}

Lower prevalence rates of ESBL producing Klebsiella however of 5.0\% was reported in Ogun (Olowe et al., 2010) and 13.8\% in Nsukka (Afunwa et al., 2011), all in Nigeria. It is difficult to explain the variations in the prevalence rates although it may not be unconnected to study design and detection techniques used in the different studies. 
Fadeyi et al., Afr. J. Infect. Dis. (2016) 10 (1): 32 - 37

\section{http://dx.doi.org/10.4314/ajid.v10i1.7}

In this study, the ratio of occurrence of Klebsiella pneumoniae to Klebsiella oxytoca was approximately 3:1. This agrees with de la Torre et al. (de la Torre et al., 1985 ) who had stated that Klebsiella infections are caused mainly by K.pneumoniae and K.oxytoca in the ratio of 4:1. This finding may be related to the pathogenic potentials and hence medical importance of these species of Klebsiella compared with others (Podschun and Ullmann, 1998).

The rate of isolation of ESBL producing Klebsiellae was highest from infected wounds (24\%) in this study. This contrasted with the findings of Okesola and Oni (2012), and Olowe et al (2012) who reported $42.1 \%$ and $24.3 \%$ of EPK isolation rates respectively from sputum indicating the lungs as the major infected organ source of the organism in their own studies. The reason for this deviation is difficult to decipher, hence further studies will be needed to clarify this issue.

As found in this study, 75\% of Klebsiellae from the Intensive Care Unit (ICU) in our hospital were EPK. Shanfayar et al. (2010) had documented a high (100\%) ESBL producing Klebsiella isolation rates from the ICU. ESBL producing organisms can easily spread among ICU patients via the hands of health care practitioners and hospital equipment (Bermudes et al., 1995). In the same light, Fillipa et al (2013) documented an outbreak of ESBL producing bacteria among ICU patients in France whose origin was traced to a patient transferred to the ICU from Nigeria. These findings suggest the need for special infection control measures and antibiotics usage in our ICUs.

ESBL producing Klebsiella organisms in this study were multi-drug resistant. Most common locally available antimicrobial were inactive against EPK given their resistance levels: cefotaxime (100\%), ceftazidime (94\%), trimethroprin-sulphamethoxazole (92\%), gentamicin (70\%), ciprofloxacin $(70 \%)$ and amoxicillin-clavulanate $(66 \%)$. Characteristically peculiar to multi-drug resistant organisms, the ESBL producing Klebsiella in this study were not only resistant to $\beta$-lactam drugs but non- $\beta$-lactam antibiotics like the aminoglycosides and fluoroquinolones as well (Okonko et al., 2009a). The high rates of resistance to antibiotics have been linked to the pressure of antibiotic usage. This has selective effect on resistant strains and promotes its spread. The multi-drug resistant organisms are now major public health concern as they limit the available drugs that can be used to treat infections (Pitout and Laupland, 2008).

Although cefepime showed considerable activity against ESBL producing Klesiellae in this study, CLSI (2013) recommends that in-vitro results of all penicillins and cephalosporins should be reported as resistant for ESBL isolates of E.coli and Klebsiella species irrespective of their individual test results. Therefore, cefepime may not demonstrate reasonable therapeutic effect in-vivo and should be used with caution in the management of ESBL producing Klebsiellae infections in our area where poverty could impair the use of the proven and recommended carbapenems due to cost.

All the EPK examined in this study were sensitive to doripenem with only $2 \%$ displaying intermediate susceptibility to imipenem. Carbapenems therefore remain the best treatment option for infections caused by ESBL producing Klesiellae in our setting as elsewhere. One possible explanation for the perceived activity of the carbapenems in our locality is its late arrival in the Nigerian market. Ensuring its continued relevance as a treatment option for the Nigerian patient hence will require a coordinated rational usage given the emergence of carbapenemases in some regions of the world. Judicious use of carbapenems should be a composite part of a functional antibiotic prescription policy which is lacking in most African countries to avoid treatment failures

\section{Conclusion}

The prevalence of EPK in this study is high and they are generally multi-drug resistant. Carbapenems however remain the most useful therapy for the infections caused by these organisms. A coordinated rational usage of carbapenems and functional antibiotic prescription policy is desired to avoid treatment failures. Continuous surveillance through well-equipped laboratories for prompt accurate detection and reporting of EPK as well as identification of multi-drug resistance should equally become a routine in our hospitals.

\section{$\bullet$}

Conflict of Interest: The authors declare that they have no competing interests.

Acknowledgement: We thank Mr Afolabi and Mrs Remi for preparing all the media used in carrying out the study.

\section{References}

1. Afunwa RA, Odimegwu DC, Iroha RI, Esimone CO. (2011). Antimicrobial resistance status and prevalence rates of extended spectrum beta-lactamase (ESBL) producers isolated from a mixed human population. Bosnian Journal of Basic Medical Sciences, 11 (2):91-6

2. Akande T.M., Ologe M, and Medubi G.F. (2009). Antibiotic prescription pattern and cost at the University of Ilorin Teaching hospital, Ilorin, Nigeria. International Journal of Tropical Medicine, 4(2): 50-54.

3. Bermudes H, Arpin C, Jude F, El-Harrif Z, Bébéar C, Quentin C. (1997). Molecular epidemiology of an outbreak due to extended-spectrum beta-lactamase-producing enterobacteria in a French hospital. European Journal of Clinical Microbiology and Infectious Diseases, 16:523529.

4. Bradford, P.A. (2001). Extended spectrum $\beta$-lactamases in the 21st century: Characterization, epidemiology and detection of this important resistance threat. Clinical Microbiology Review,14: 933-951

5. Cheesebrough M. (2006). District Laboratory Practice in Tropical Countries, Part 2. Cambridge University Press. P.187.

6. CLSI. Performance Standards for Antimicrobial Susceptibility Testing; (2013). Twenty second International Supplement. CLSI document M100-S22 Vol. 32 No. 3. Wayne PA: Clinical Laboratory Standard Institute. Pp.46-52

7. de la Torre, M. G., J. Romero-Vivas, and J. Martinez-Beltràn.(1985). Klebsiella bacteremia: An analysis of 100 episodes. Review of Infectious Diseases, 7:143-150 
Fadeyi et al., Afr. J. Infect. Dis. (2016) 10 (1): 32 - 37

http://dx.doi.org/10.4314/ajid.v10i1.7

8. Ejikeugwu C, Ikegbunam M, Ugwu C, Eze P, Iroha I, Esimone C. (2013). Phenotypic Detection of Klebsiella pneumoniae Strains Producing Extended Spectrum $\beta$-Lactamase (ESBL) Enzymes. Schorlarly Academic Journal of Biosciences, 1(1): 20-23.

9. Filippa N, Carricajo A, Grattard F, Fascia P, Sayed FE, Defilippis JP, et al. (2013). Outbreak of multi drug resistant Klebsiella pneumoniae carrying qnrB1 and blaCTX M15 in a French intensive care unit. Annals of Intensive Care Unit, 3:18.

10. Knothe H, Shah P, Kremery V. (1983). Transferable resistance to cefotaxime, cefoxitin, cefamandole and cefuroxime in clinical isolates of Klebsiella pneumoniae and Serratia marcescens. Infection, 11:315-317

11. Okesola AO, Oni AA. (2012). Prevalence of Extended Spectrum Beta Lactamase producing Klebsiella in a tertiary care Hospital in south west Nigeria. International Journal of Pharmaceutical and Biomedical Sciences, 3(4): 148-151.

12. Okonko IO, Soleye FA, Amusan TA, Ogun AA, Ogunnisi TA, Ejembi J. (2009a). Incidence of Multi- Drug Resistance (MDR) organisms in Abeokuta, southwestern Nigeria. Global Journal of Pharmacy, 3(2):69-80

13. Olowe O.S., Aboderin B.W. Motayo B.O, Ibeh O, Adegboyega T.T, and Ogiowa I.J. (2010). Detection of Extented Spectrum BetaLactamase producing strains of Escherichia coli and Klebsiella species in a tertiary health center in Ogun state, Nigeria. International Journal Tropical Medicine, 5(3):62-64

14. Olowe OA, Oladipo GO, Makajuola OA, Olaitan JO. (2012). Prevalence of extended Spectrum Beta-Lactamases(ESBLs) carrying genes in klebsiella spp. from clinical samples at Ile-ife, South Western Nigeria. International Journal of Pharma Medicine and Biological Sciences, 1(2): 129-138.

15. Osazuwa F, Osazuwa EO. (2013). Detection of extended spectrum beta-lactamase producing Klebsiella pneumoniae and their susceptibility rates to antibiotics in University of Benin Teaching Hospital, Benin City, Nigeria”. Research Journal of Pharmaceutical and Biochemical Sciences, 2(1);603-607

16. Paterson D, Bonomo R. (2005). Extended-spectrum $\beta$-lactamases: a clinical update. Clinical Microbial Review,18: 657-86.

17. Paterson DL, Ko WC, Gottberg AV. (2003). Antibiotic therapy for Klebsiella pneumoniae bacteraemia: implications of production of extended-spectrum beta-lactamases. Clinical Infectious Diseases, 39:31-37.

18. Paterson, D. L. (2000). Recommendation for treatment of severe infections caused by Enterobacteriaceae producing extended-spectrum $\beta$ lactamases (ESBLs). Clinical Microbial Infections, 6:460- 463.

19. Pitout, J.D.D and Laupland K.B. (2008). Extended-spectrum $\beta$-lactamase-producing enterobacteriaceae: An emerging public-health concern. Lancet Infectious Diseases, 8: 159-166.

20. Podschun R, Ullmann U. (1998). Klebsiella spp. as nosocomial pathogens: epidemiology, taxonomy, typing methods, and pathogenicity factors. Clinical Microbiology Review,11:589-603.

21. Rodriquez-Bano J, Navarro MD, Romero L, Martinez-Martinez L, Muniain MA and Perea EJ. (2004) Epidemiology and clinical features of infections Caused by extended spectrum beta-lactamase producing Escherishia coli in non Hospitalized patients. Journal of Clinical Microbiology, 42: 1089-1094.

22. Schwaber M, Navon-Venezia S, Kaye K, Ben-Ami R, Schwartz D and Carmeli Y. (2006). Clinical and Economic Impact of Bacteremia with Extended-Spectrum- Lactamase-Producing Enterobacteriaceae. Antimicrobial Agents and Chemotherapy, 50(4):1257- 1262

23. Shayanfar N, Rezaei M, Ahmadi M, Ehsanipour F. (2010). Evaluation of extended spectrum betalactamases (ESBL) positive strains of Klebsiella pneumoniae and Escherichia coli in bacterial cultures. Iranian Journal of Pathology, 5(1): 34-39 